\title{
Propriedades mecânicas de compósitos cimentícios produzidos com lodo de estação de tratamento de efluentes da indústria de batata pré-fritas
}

\author{
Mechanical properties of cement composites \\ produced with sludge from the pre-fried \\ potato industry's effluent treatment station
}

Domingos Sávio de Resende

Centro Federal de Educação Tecnológica de Minas Gerais - CEFET-MG

Coordenação de Eletrônica, Campus IV savio@araxa.cefetmg.br

Augusto Cesar da Silva Bezerra Centro Federal de Educação Tecnológica de Minas Gerais - CEFET-MG

Coordenação de Edificações, Campus IV augustobezerrra@araxa.cefetmg.br

Antonio Maria Claret de Gouveia Universidade Federal de Ouro Preto

Ouro Preto, Brasil.

claretgouveia@uol.com.br

\section{Resumo}

O grande volume de resíduos sólidos industriais gerados pela indústria de batatas pré-fritas e o alto custo do seu manejo estimulam pesquisas em busca de soluções mais adequadas para a sua destinação final. Esse trabalho caracterizou o resíduo sólido (lodo) de Estação de Tratamento de Efluentes (ETE) de indústria de batatas. O lodo foi processado através de secagem, desidratação, moagem e peneiramento. Para a caracterização do lodo foram utilizadas técnicas de granulometria a laser, microscopia eletrônica de varredura (MEV) com espectrômetro de raios X dispersivo em energia (EDS), difração de raio X, análise térmica diferencial (DTA), termogravimétrica (TGA) e perda ao fogo. Após a caracterização, o lodo foi utilizado para a confecção de compósitos cimentícios, em substituição parcial do cimento, em massa equivalente para substituição do mesmo volume, determinado pelas massas específicas de ambos materiais, nas porcentagens de 3, 5, 7 e 10\%. Os compósitos foram ensaiados para a obtenção da resistência à compressão e do módulo de elasticidade dinâmico. A utilização do lodo de ETE como pozolana foi descartada, tendo-se como base o resultado do ensaio de perda ao fogo. Nos ensaios mecânicos realizados, nota-se que a adição de lodo, em substituição parcial ao cimento, impactou, negativamente, de forma mais relevante a resistência à compressão. Tais ensaios foram considerados satisfatórios para os compósitos com substituição do cimento por 3\% e 5\% de lodo.

Palavras-chave: Lodo de ETE, indústria de batatas pré-fritas.

\begin{abstract}
The large volume of industrial solid waste generated by the pre-fried potato industry and the high cost of its management encouraged researchers to find solutions best suited to its final destination. This study characterized the solid residue (sludge) from the effluents pre-fried potatoes industry's treatment station. The sludge was processed by drying, grinding and sieving. Laser particle size, scanning electron microscopy (SEM) with X-ray spectrometer dispersive energy (EDS), X ray diffraction, differential thermal analysis (DTA), thermogravimetric (TGA) and loss on ignition were used to characterize the sludge. After characterization, cement was made using 3, 5, 7 and
\end{abstract}


10 percentages of the sludge to replace the cement. The composites were tested to obtain the compressive strength and dynamic elasticity modulus. Based on the result of the loss on ignition, the use of sludge as pozzolan was discarded. Based on the mechanical property tests carried out, it was noted that the addition of sludge as a partial replacement of cement is negatively impacted, with only the results of the cement composites of $3 \%$ and $5 \%$ being satisfactory.

Keywords: Sludge, pre-fried potato industry, cement composites.

\section{Introdução}

A indústria alimentícia instalada no município de Araxá (MG) é geradora de um grande volume de resíduos sólidos originado no processo de industrialização da batata. O resíduo industrial gerado não pode ser depositado em aterros sanitários, por isso faz-se necessário desenvolver novas técnicas de reutilização desse material. Além disso, o lodo da estação de tratamento de esgoto da indústria de batatas pré-fritas possui características que favorecem o seu reaproveitamento. Entre os fatores que contribuem para o seu reaproveitamento estão o grande volume gerado em um ponto fixo de emissão, a ausência de sazonalidade na geração, a não periculosidade do resíduo, a ausência de contaminação com esgoto sanitário por ser gerado em uma linha industrial.

A produção de batatas pré-fritas no município, no ano de 2009, representou

\section{Materiais e métodos}

O lodo utilizado nessa pesquisa, foi coletado na estação de tratamento de esgoto da empresa BEM BRASIL ALIMENTOS S/A. Essa estação recebe somente efluentes do processo industrial. $\mathrm{O}$ resíduo passou por um processo de desidratação, onde todo o material foi exposto ao sol para que fosse eliminada uma parcela da umidade. Antes da exposição do lodo ao sol, foram coletadas 3

\section{Caracterização do lodo}

Para determinação das dimensões das partículas, foi realizado, ensaio de granulometria a laser no equipamento CILAS 1160, utilizando-se como meio dispersor, água destilada.

O ensaio, para a determinação da massa específica do lodo, foi realizado adicionando-se $250 \mathrm{ml}$ de água ao frasco de Le Chatelier, de forma que o líquido ficasse na marca de $0 \mathrm{ml}$ visível na graduação do tubo. Em seguida, secou-se o colo dos frascos com papel absorvente e
$28 \%$ do mercado nacional. Atualmente são processadas seis mil toneladas de batatas por hora e há previsão de se processarem quinze mil toneladas por hora, o que projeta um grande aumento na produção de lodo pela estação de tratamento de esgoto da linha industrial. A primeira destinação natural do lodo de estações de tratamento de efluentes da indústria das batatas pré-fritas seria como fertilizante, no entanto seu longo tempo de compostagem e a baixa porcentagem de nutrientes presentes inviabilizam tal uso.

A produção de cimento é um dos maiores contribuintes para as emissões globais de carbono (Huntzinger \& Eatmon, 2009). Atualmente, com o aumento da pressão para se reduzir a poluição ambiental, a indústria de cimento começou a adotar uma série de métodos para alcançar essas metas (Zhang \& Wang, 2010).

Lodos de estações de tratamento

amostras para a determinação do teor de umidade do material. As amostras foram mantidas em estufa em uma temperatura entre 60 e $65^{\circ} \mathrm{C}$, até a obtenção da constância de massa. A umidade foi calculada através da razão entre a massa de água e a massa seca do lodo.

Após a exposição ao sol, o material foi colocado em estufas com temperatura entre 60 e $65^{\circ} \mathrm{C}$. Essa temperatura foi es-

foram feitas as devidas leituras de volume novamente. Foram pesados $35 \mathrm{~g}$ de lodo e a amostra foi colocada dentro do frasco, com auxílio de um funil. Logo, foram feitas novas leituras de volume do frasco e foi calculada a variação de volume da água contida no recipiente após $15 \mathrm{mi}$ nutos. O ensaio foi repetido mais duas vezes e desprezado aquele que mais se afastou da média. A massa específica foi obtida dividindo-se a massa do material introduzida pela diferença de volume do de água (ETA) já foram estudados como adição em compósitos cimentícios, sejam argamassas ou concretos (Sales et al., 2010; Sales et al., 2011; Sales \& Souza, 2009). Já existem várias pesquisas sobre a utilização de lodo de ETA ou de ETE incinerado como adição mineral em compósitos cimentícios (Chen et al., 2011; Fontes, 2003; Geyer, 2001). Não foram encontradas pesquisas sobre a utilização de lodo de estação de tratamento de esgoto da indústria de batatas pré fritas in natura e poucos trabalhos foram publicados sobre a utilização de lodo de estação de tratamento de esgoto não incinerado (in natura). Visando a ampliar o conhecimento sobre as possibilidades do uso do lodo de estação de tratamento de esgoto da indústria de batata pré-fritas, esse trabalho avaliou as características físicoquímicas do lodo e, avaliando, também, sua aplicação em compósitos cimentícios.

colhida com o objetivo de não se eliminar a matéria orgânica, caso estivesse presente no lodo. O lodo foi mantido na estufa até a obtenção da constância de massa. Em seguida, o material foi britado para redução da granulometria e uniformização. Após o processo de britagem, o resíduo foi moído para maior redução das partículas. O lodo utilizado foi o passante na peneira com abertura de $0,150 \mathrm{~mm}$.

recipiente utilizado.

A difração de raios $\mathrm{X}$ (DRX) foi utilizada para se verificar a presença de fases cristalinas. A identificação das fases presentes foi baseada na comparação de um perfil desconhecido com o conjunto de difração-padrão coletado e mantido pelo JCPDS (Joint Committee on Powder Diffraction Standards). A análise via difração de raio $X$ foi feita utilizando-se um tubo de cobalto acoplado ao equipamento Miniflex da marca Rigaku. 
A análise via microscopia eletrônica de varredura (MEV) foi realizada utilizando-se equipamento Stereoscam-200 da Cambridge. As amostras do lodo foram espalhadas sobre uma fita de carbono previamente colada a um porta-amostra. Em seguida, foi realizada a metalização, que consistiu na precipitação de uma película de material condutor (carbono) sobre a superfície da mesma, possibilitando a condução de corrente elétrica. Foram realizadas análises de composição química semiquantitativas em diferentes regiões da amostra com espectrômetro de raios $\mathrm{X}$ dispersivo em energia.

A análise térmica diferencial (DTA) é uma técnica calorimétrica, que registra a temperatura e o fluxo de calor associado com as transições térmicas em um material e foi utilizada para verificação dos eventos endotérmicos e exotérmicos. O equipamento utilizado foi o modelo DTG-60H com capacidade para análise diferencial e termogravimétrica de modo simultâneo (TGA/DTA) da marca Shimadzu.

A perda ao fogo $(\% \mathrm{PF})$ consistiu em colocar a amostra de $1,000 \mathrm{~g}(+/-0,001 \mathrm{~g})$ em um cadinho de porcelana e calcinar em forno mufla à temperatura de $950^{\circ} \mathrm{C}\left(+/-50^{\circ} \mathrm{C}\right)$, por $20 \mathrm{~min}$. Esperou-se a amostra esfriar e a mesma foi pesada após a calcinação. $\mathrm{O}$ valor de perda ao fogo foi obtido através da relação entre a perda de massa no processo de calcinação e a massa inicial da amostra.

\section{Confecção e avaliação dos compósitos cimentícios}

Para a avaliação da utilização do lodo como adição em concretos, foram confeccionados 6 corpos de prova para cada compósito cimentício com substituição parcial do cimento pelo lodo nos percentuais e quantidades apresentados na Tabela 1.

Apesar de a demanda de água, para a trabalhabilidade requerida pela norma NBR 5752 (ABNT, 1992), ser obtida pelo espalhamento de $225+/-5 \mathrm{~mm}$, optou-se por fixar a relação água cimento em 0,7, tomando-se, como base, a quantidade de cimento inicial do traço de referência $(0 \%$ de lodo). A quantidade de lodo foi calculada em massa, para a substituição ser realizada no mesmo volume, dividindo-se a massa específica do lodo $\left(1,231 \mathrm{~g} / \mathrm{cm}^{3}\right)$ pela massa específica do cimento Portland $\left(3,081 \mathrm{~g} / \mathrm{cm}^{3}\right)$. O resultado foi multiplicado pela massa do cimento retirada, massa esta referente ao percentual de

Tabela 1

Proporção de materiais utilizados para confecção dos compósitos cimentícios.

\section{Resultados e discussões}

Na Tabela 2, são apresentados os diâmetros equivalentes das partículas do lodo através do método de granulometria a laser.

A granulometria a laser indica que o lodo de esgoto de ETE utilizado atingiu valores muito próximos ao estabelecido pela NBR 12653 (ABNT, 1992), que define um limite mínimo de finura para as pozolanas substituição. A quantidade de cimento e a quantidade de areia utilizadas na composição do traço com $0 \%$ de lodo (referência) foram obtidas através da NBR 7215 (ABNT, 1996) para a determinação da resistência à compressão do cimento Portland. As areias foram normalizadas de acordo com a NBR 7214 (ABNT, 1982). A argamassa foi preparada por meio de um misturador mecânico e compactada, manualmente, em um molde cilíndrico de $5 \mathrm{~cm}$ de diâmetro por $10 \mathrm{~cm}$ de altura, por um procedimento normalizado. A moldagem dos corpos de prova foi feita imediatamente após o amassamento e com a maior rapidez possível. Os corpos de prova foram desmoldados no dia seguinte e submetidos à cura em água saturada de cal até a data de ruptura.

A avaliação do comportamento dos compósitos cimentícios confeccionados, para análise da influência da variação dos teores de lodo de ETE de indústria de batatas em substituição ao cimento Portland $(0 \%, 3 \%, 5 \%, 7 \%$ e $10 \%)$, foi realizada aos 28 dias por meio de ensaios de determinação da resistência à compressão e determinação do módulo de elasticidade dinâmico. Para a determinação do módulo de elasticidade dinâmico, por frequência ressonante forçada, foi utilizado o equipamento Erudite MKII. Foram pesados 3 corpos de prova de cada traço, sendo que tais corpos de prova foram submetidos à variação de frequência forçada de $15 \mathrm{KHz}$ a $25 \mathrm{KHz}$. Para cada corpo de prova foram realizadas 5 leituras da frequência ressonante. Foram totalizadas 15 leitura para cada traço. Após o ensaio de frequência ressonante, os corpos de prova foram capeados com enxofre e rompidos para a determinação da resistência à compressão. Foram ensaiados a compressão 6 corpos de prova para cada traço.

\begin{tabular}{c|c|c|c|c|c}
\hline \multicolumn{7}{c}{ Quantidade utilizada em gramas nos compósitos (g) } \\
\hline Materiais & $\mathbf{0 \%}$ lodo & $\mathbf{3 \%}$ lodo & $\mathbf{5 \% ~ l o d o}$ & $\mathbf{7 \%}$ lodo & $\mathbf{1 0 \%}$ lodo \\
\hline Cimento & 624,00 & 605,28 & 592,80 & 580,32 & 561,60 \\
\hline Lodo & 0,00 & 7,48 & 12,46 & 17,45 & 24,93 \\
\hline Água & 436,80 & 436,80 & 436,80 & 436,80 & 436,80 \\
\hline Areia normal & \multicolumn{7}{|c}{} \\
\hline Fração grossa & 468,00 & 468,00 & 468,00 & 468,00 & 468,00 \\
\hline Fração média grossa & 468,00 & 468,00 & 468,00 & 468,00 & 468,00 \\
\hline Fração média fina & 468,00 & 468,00 & 468,00 & 468,00 & 468,00 \\
\hline Fração fina & 468,00 & 468,00 & 468,00 & 468,00 & 468,00 \\
\hline
\end{tabular}

artificiais tipo E, em que o percentual máximo retido na peneira $45 \mu \mathrm{m}$ deve ser de $34 \%$. O lodo utilizado, de acordo com a granulometria a laser, possui mais de $90 \%$ dos seus grãos inferiores a $62,78 \mu \mathrm{m}$ e tamanho médio de $32,29 \mu \mathrm{m}$.

O material se apresentou amorfo e não foi detectada a presença de fase cris- talina. Foi feita a calcinação da amostra a $300^{\circ} \mathrm{C}$ por 2 horas, na tentativa de se obter uma estrutura cristalina da mesma, porém o difratograma apresentado na Figura 1 mostra que o material manteve-se com grande grau de amorfismo, apresentando somente um pico de cristalinidade de baixa intensidade. 


\begin{tabular}{c|c}
\hline Diâmetro a 10\% (d10) & $4,78 \mathrm{~mm}$ \\
\hline Diâmetro a $50 \%(\mathrm{~d} 50)$ & $29,80 \mathrm{~mm}$ \\
\hline Diâmetro a 90\% (d90) & $62,78 \mathrm{~mm}$ \\
\hline Diâmetro médio & $32,29 \mathrm{~mm}$ \\
\hline
\end{tabular}

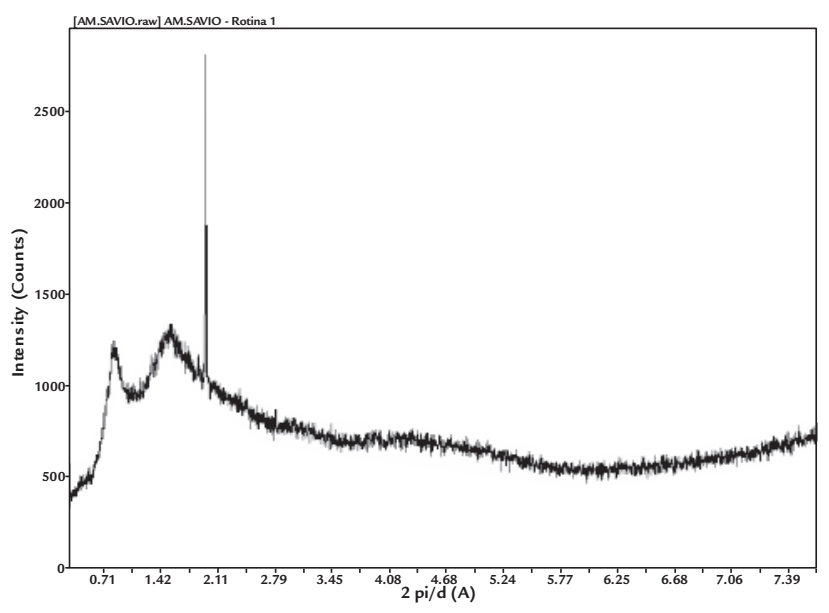

A microscopia eletrônica de varredura foi realizada juntamente com o espectrômetro de raios $\mathrm{X}$ dispersivo em energia (EDS), para a determinação da

composição química semi-quantitativa por amostragem pontual. Foi detectada a presença de $\mathrm{Mg}, \mathrm{Al}, \mathrm{Si}, \mathrm{P}, \mathrm{S}, \mathrm{Cl}, \mathrm{K}, \mathrm{Ca}, \mathrm{Ti}$ e Fe, conforme apresentado na Tabela 3.

\begin{tabular}{c|c}
\hline Elemento & Teor \% (em massa) \\
\hline $\mathrm{Mg}$ & $1,8 \pm 0,1$ \\
\hline $\mathrm{Al}$ & $13,9 \pm 0,6$ \\
\hline $\mathrm{Si}$ & $11,0 \pm 0,3$ \\
\hline $\mathrm{P}$ & $13,1 \pm 0,1$ \\
\hline $\mathrm{S}$ & $12,3 \pm 0,4$ \\
\hline $\mathrm{Cl}$ & $5,5 \pm 0,9$ \\
\hline $\mathrm{K}$ & $18,5 \pm 0,5$ \\
\hline $\mathrm{Ca}$ & $11,4 \pm 0,4$ \\
\hline $\mathrm{Ti}$ & $2,2 \pm 0,2$ \\
\hline $\mathrm{Fe}$ & $10,2 \pm 0,7$ \\
\hline
\end{tabular}

de alumínio e de cloro é justificada pela adição de floculantes no processo de obtenção do lodo antes do tanque de decantação primária na estação de tratamento de esgotos da indústria de batatas.

Na Figura 2, são apresentadas as micrografias do lodo. Percebe-se que a morfologia do lodo indica que o material apresenta-se compacto e sem estrutura lamelar, o que indicaria uma baixa área superficial específica.

$\mathrm{Na}$ Figura 3, estão representadas as curvas de DTA e TGA da amostra de lodo sob condição de atmosfera inerte (nitrogênio). A análise da curva DTA demonstra os eventos endotérmicos e exotérmicos ocorridos com a amostra. A curva TGA indica as perdas de massa ocorridas durante o processo. Por volta de $80^{\circ} \mathrm{C}$, ocorre um evento endotérmico, com pequena perda de massa. Tal evento, provavelmente, está relacionado à decomposição de substâncias protéicas e/ou

de polissacarídeos presentes na amostra, visíveis na curva de TGA. Já entre $170^{\circ} \mathrm{C}$ e $500^{\circ} \mathrm{C}$, a curva de DTA mostra um evento exotérmico de média intensidade, acompanhado de grande perda de massa. Esse evento deve estar relacionado à oxidação do material. Entre $500^{\circ} \mathrm{C}$ e $1120^{\circ} \mathrm{C}$, ocorre uma série de eventos endotérmicos, que podem estar relacionados a processos de fusão ou de transição de fases e às reações de decomposição ou à dissociação das várias substâncias presentes na amostra. Com relação a tais substâncias, não podemos afirmar com precisão a sua identidade, por se tratar de amostra de natureza complexa, podendo apenas afirmar que eventos em menores temperaturas, provavelmente, se relacionam a substâncias de natureza orgânica, enquanto eventos em maiores temperaturas, geralmente, são atribuídos a material de natureza inorgânica. $\mathrm{O}$ resultado percentual da perda ao fogo médio obtido
Tabela 2

Diâmetros equivalentes através da granulometria a laser das partículas da amostra passante na peneira com abertura de $0,150 \mathrm{~mm}$.

Figura 1

Difratograma representando o sólido amorfo para a amostra de resíduo passante na peneira com abertura de $0,150 \mathrm{~mm}$.

Os elementos químicos encontrados, na maioria, são constituintes da batata, ou estavam presentes na casca como restos de adubação química. A presença

Tabela 3

Resultados da composição

química do lodo. foi de $94,75 \%$, o que indicaria grande percentual de matéria.

Os resultados de resistência à compressão são apresentados na Figura 4. A análise dos resultados mostra que as variações encontradas estão ligadas ao teor de adição de lodo de ETE, em substituição parcial ao cimento. Encontrou-se uma relação inversamente proporcional, na qual, quanto maior o consumo de lodo no compósito, menor foi a resistência encontrada. Em relação ao compósito 1 ou de referência ( $0 \%$ de lodo), ocorreu a redução da resistência à compressão nas seguintes proporções: compósito 2 (3\% de lodo) alcançou $-32 \%$ da resistência à compressão; compósito 3 ( $5 \%$ de lodo), -41\%; compósito 4 (7\% de lodo), - $47 \%$ e compósito 5 (10\% de lodo), $-59 \%$.

$\mathrm{Na}$ Figura 5, são apresentados os resultados de módulo de elasticidade dinâmico obtido pelo ensaio de frequência ressonante forçada. Verificou-se, nos re- 
sultados dos corpos de prova que as adições de teores de lodo de ETE de 3\%, $5 \%, 7 \%$ e de $10 \%$, comparadas aos teores das argamassas de referências $(0 \%$ de lodo de ETE), impactaram, de forma relevante, a redução dos valores médios encontrados. A variação percentual média encontrada foi de $-20 \%$, para os compósitos com teor de adição de 3\%, de $-25 \%$, para o teor de $5 \%$, de $-33 \%$ para o teor de adição de $7 \%$ e de $-42 \%$, para $10 \%$.
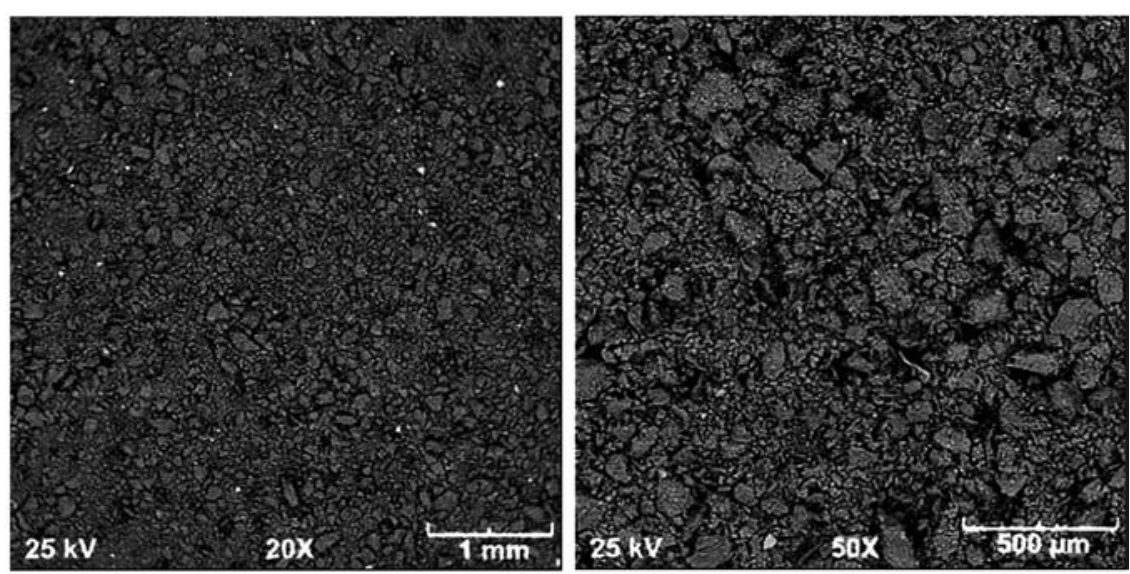

Figura 2 Micrografia do lodo.
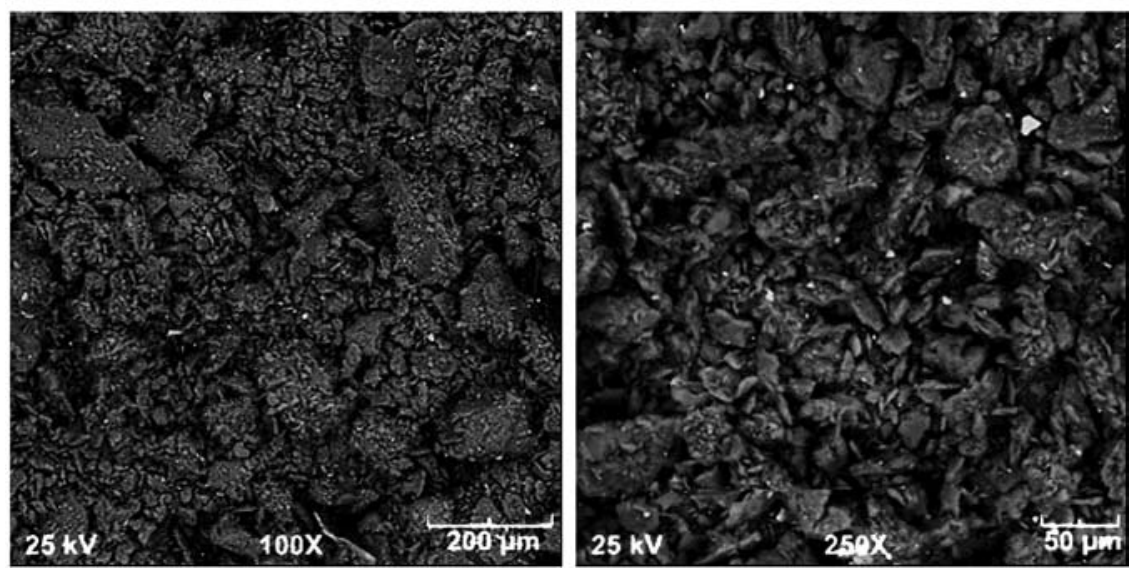

Figura 3 Curvas resultante da análise térmica DTA e TGA.
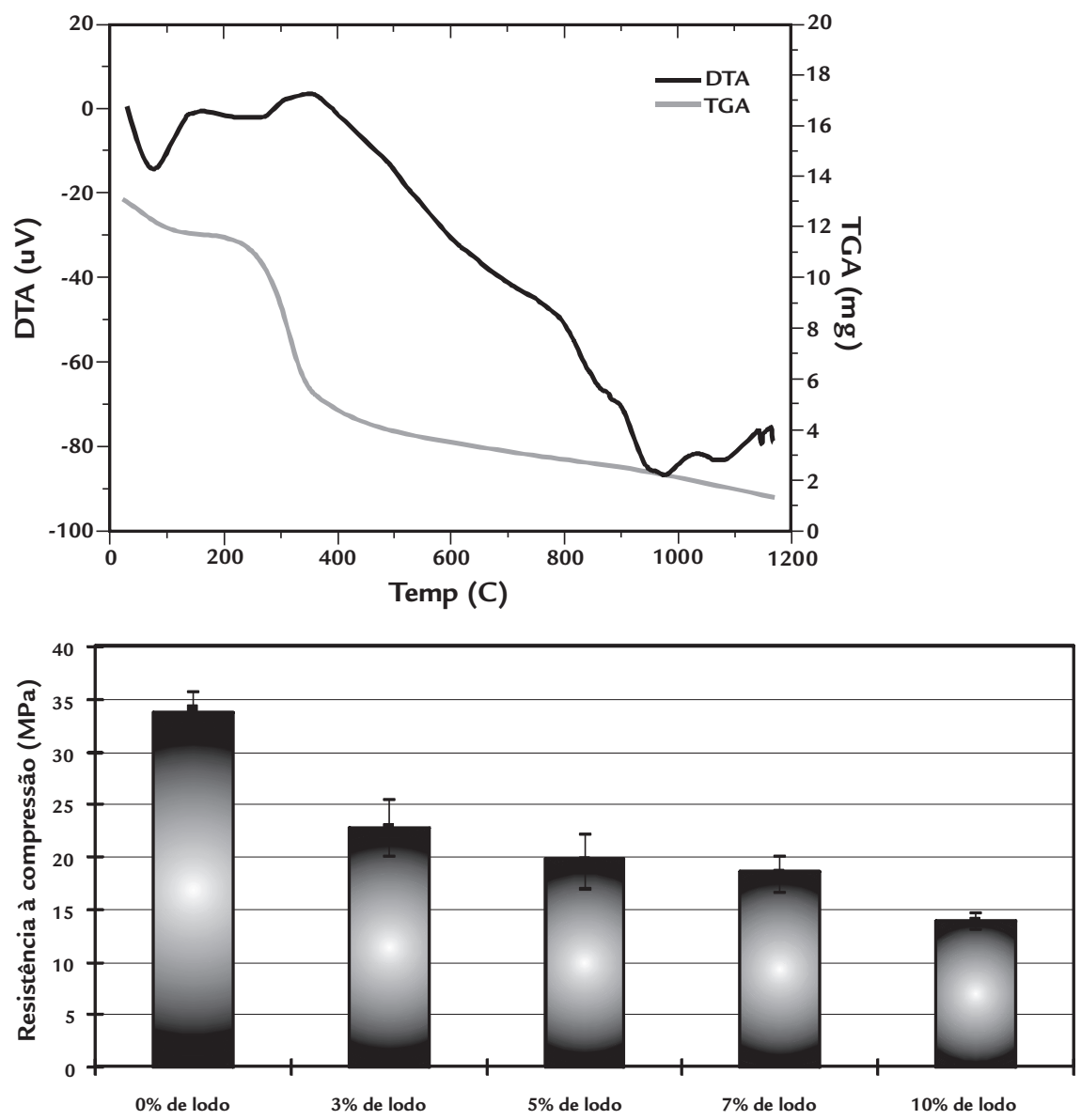


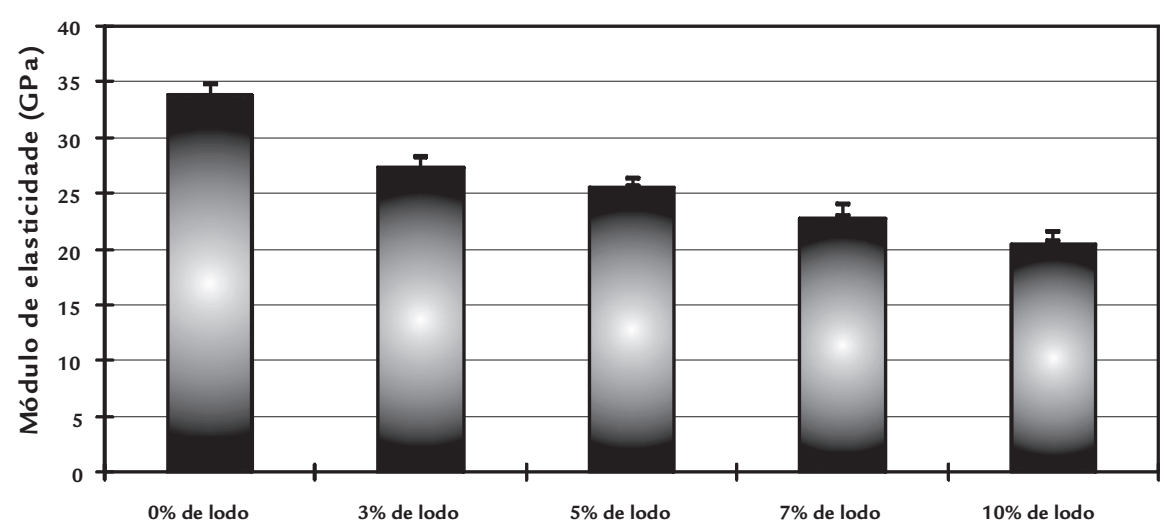

\section{Conclusão}

O resíduo analisado apresentou, inicialmente, características físicas e químicas que indicavam a possibilidade de utilização em compósitos cimentícios, pois o material, na difratometria de raio $\mathrm{X}$ apresentou-se parcialmente amorfo, o que pode indicar uma possibilidade de reação pozolânica. A morfologia do lodo indicou um material compacto e sem estrutura lamelar, que, comparado a um material de mesma granulometria, apresentaria uma menor área superficial específica. A utilização do lodo de ETE, como pozolana tipo E, conforme a NBR 12653 (ABNT, 1992), foi descartada, tendo-se, como base, o resultado do en-
Figura 5

Resultados de módulo de elasticidade dos compósitos.

\section{Referências bibliográficas}

ASSOCIAÇÃO BRASILEIRA DE NORMAS TÉCNICAS. NBR 5752 - Materiais pozolânicos - determinação da atividade pozolânica com cimento Portland indice de atividade pozolânica com cimento. Rio de Janeiro, 1992a.

ASSOCIAÇÃO BRASILEIRA DE NORMAS TÉCNICAS. NBR 7214 - Areia normal para ensaio de cimento. Rio de Janeiro, 1982.

ASSOCIAÇÃO BRASILEIRA DE NORMAS TÉCNICAS. NBR 7215 - Cimento Portland - determinação da resistência à compressão - método de ensaio. Rio de Janeiro, 1996.

ASSOCIAÇÃO BRASILEIRA DE NORMAS TÉCNICAS (ABNT) - NBR 12653 materiais pozolânicos - especificação. Rio de Janeiro, 1992b.

CHEN, J. H., HUANG, J. S., CHANG, Y. W. Use of reservoir sludge as a partial replacement of metakaolin in the production of geopolymers. Cement and Concrete Composites, v. 33, n. 5, p. 602-610, May 2011.

FONTES, C. M. A. Potencialidades da cinza de lodo de estações de tratamento de esgotos como material suplementar para a produção de concretos com cimento Portland. Rio de Janeiro: Universidade Federal do Rio de Janeiro, 2003. (Tese de Doutorado).

GEYER, A. L. B. Contribuição ao estudo da disposição final e aproveitamento da cinza de lodo de estações de tratamento de esgotos sanitários como adição ao concreto. Porto Alegre: Universidade Federal do Rio Grande do Sul, 2001. (Tese de Doutorado).

HUNTZINGER, D., EATMON, T. A life cycle assessment of portland cement manufacturing: comparing the traditional process with alternative technologies. Journal of Cleaner Production, n. 17, p. 668-675, 2009.

SALES, A., SOUZA, F. R. Concretes and mortars recycled with water treatment sludge and construction and demolition rubble. Construction and Building Materials, v. 23, n. 6, p. 2362-2370, 2009.

SALES, A., SOUZA, F. R., SANTOS, W. N., ZIMER, A. M., COUTO, F. A. R. Lightweight composite concrete produced with water treatment sludge and sawdust: thermal properties and potential application. Construction and Building Materials, n. 24, p. 2446-2453, 2010.

SALES, A, SOUZA, F. R., ALMEIDA, F. C. R. Mechanical properties of concrete produced with a composite of water treatment sludge and sawdust. Construction and Building Materials, v. 25, n. 6, p. 2793-2798, June 2011.

ZHANG, J., WANG, Y. Low carbon economy in the cement industry. In: CHINA INTERNATIONAL CEMENT CONFERENCE. Proceedings... 2010 (in Chinese).

saio de perda ao fogo. Nos ensaios mecânicos realizados, nota-se que a adição de lodo, em substituição parcial ao cimento, impactou, de forma mais relevante, a resistência à compressão. Tais ensaios foram considerados satisfatórios para os compósitos com substituição de 3\% e $5 \%$ de cimento. 